\title{
LAS OBRAS DE DEFENSA \\ CONTRA INUNDACIONES \\ EN LA FRONTERA NORTE DE MÉXICO: EVOLUCIÓN DE UN RAMO DE OBRAS PÚBLICAS (I 879-I 944)
}

The Defensive works Against Floods in MeXico's NORTHERN BORDER: EVOLUTION OF A PUBLIC WORKS BRANCH (I 879-I 944)

\section{ALEJANDRO GONZÁLEZ MILEA}

\section{RESUMEN}

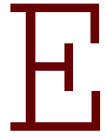

l artículo revisa discusiones de proyectos y obras de defensa contra inundaciones que se condujeron entre las décadas de 1870 y 1940 en la frontera norte. Mediante fuentes primarias puede explicarse el carácter pragmático y versátil de sus responsables —en Nogales, Ciudad Juárez y el Bajo Río Bravo-, pues se ocuparon en trabajos de hidráulica y programas y proyectos de arquitectura, e incluso aplicaron rudimentos de planificación urbana. El contexto institucional revela cómo el tránsito desde las mejoras materiales hacia una noción de obras públicas, en ramos, sucedió dentro de campos ocupacionales en disputa y una burocracia técnica heterogénea.

1 Universidad Autónoma de Ciudad Juárez. Correo: alejandro.gonzalez.m@uacj.mx. 
Palabras clave: inundaciones, obras de defensa, historia de las obras públicas, ingeniería, arquitectura

\section{ABSTRACT}

This article examines public works projects for flood control conducted between the decades of 1870 and 1940 in Mexico's Northern Border. Using primary sources it demonstrates technician's pragmatic and versatile approach -in Nogales (Sonora), Ciudad Juarez (Chihuahua) and Bajo Rio Bravo (Tamaulipas)-, performing a range of jobs including hydraulic works, architectural programs and projects, and also rudimentary applied urban planning. An institutional analysis reveals how the transition from ideas of material betterment to the paradigm of public works, and its variegated definition, occurred amidst a dispute between occupational fields and a heterogeneous technical bureaucracy.

Key words: floods, defensive works, public works history, engineering, architecture.

\section{INTRODUCCIÓN}

La frase "historia de las obras públicas" puede parecer que encierra un significado evidente: se trataría de los trabajos realizados para el beneficio común. Generalmente son impulsados y ejecutados por los gobiernos y a la vez se imbrican con la misma consolidación del Estado. En efecto, el Estado ha hecho obras públicas, pero también a través de sus obras se ha afianzado en los territorios que busca sujetar y controlar. En un ya clásico argumento, desde la década de 1990 Priscila Connolly (1999) destacó la necesidad de profundizar en el papel que en la historia de las obras públicas tuvieron varias instancias administrativas, la organización de su producción y los "sistemas argumentales" que regían la acción pública (pp. 142-143, 152). A través del tiempo ha habido obras y trabajos, que por su magnitud, no pudieron 
concluirse en corto tiempo, y así varias generaciones quedaban obligadas a finalizarlos, incluso no se planteaban discutir las razones de su necesidad; se trata, pues, de una dimensión compleja de las infraestructuras que en las últimas dos décadas han revisitado los antropólogos ocupados en la "materialidad" (Anand et al., 2018; Larkin, 2013). Este es el caso de las obras de defensa contra inundaciones, sobre todo cuando se relacionaron con los requerimientos de crecimiento de las ciudades, aunque este tipo de obras, tradicionalmente se había originado en la oportunidad de aprovechar el agua.

La clasificación de obras públicas por ramos, a través del tiempo, permite observar de manera detallada cómo se afianzaron los Estados. Parecería obvio afirmar que fueron las diversas especialidades - resultado de la evolución de las profesiones- las que explican la división entre ramos o géneros, como las obras hidráulicas, las obras de caminos y transporte, los edificios de gobierno o los monumentos conmemorativos y la planeación de ciudades. Aunque en un contexto monárquico y decimonónico, Inmaculada Aguilar (2012) ha revisado la definición de ramos de obra pública, de la mano de la definición del perfil profesional, destacando procesos que es necesario investigar: la hidráulica como una faceta de actividad del arquitecto, la importancia de los ingenieros de caminos en la institucionalización de obras públicas frente a otros ámbitos como el militar y el forestal, y las implicaciones administrativas y presupuestales en la definición de ramos de obra pública (pp. 62-63, 70).

Sin embargo, cuando la concepción de las obras y su ejecución cruzan varios regímenes políticos, esta división ya no resulta tan evidente, y se vuelve necesario examinar lo que académicos como Howard Davis (2016) denominaron “cultura de la construcción”, es decir, un sistema coordinado de conocimientos, reglas, procedimientos y hábitos que rodean el proceso de edificación en un momento y tiempo determinados, y dan forma al paisaje construido, abarcando no solamente las grandes y excepcionales "obras maestras" - que son pocas-, sino las obras de todos los días - que son las más- (pp. 5-6). En otras palabras, no fueron los lugares 
y las regiones los que produjeron su propio modo de concebir y ejecutar obras, sino las redes de individuos agrupados en campos sociales. A menudo la mirada del investigador se posiciona en los propósitos definidos y llevados adelante por grupos que mantienen una coherencia espacio-temporal, por ejemplo, para explicar la introducción del movimiento moderno en urbanismo; pero los equipos que emprendieron y vigilaron obras públicas, sobre todo las de gran dimensión, no siempre tuvieron esta coherencia, pues los trabajos cruzaron varias generaciones. Connolly (1999) señaló que la Revolución en México —ese movimiento armado de inicios del siglo XX que transformó instituciones- de ninguna manera quebró los procesos de producción de obras urbanas de décadas anteriores (pp. 145-146).

Pasar los anteriores argumentos al plano de las obras hidráulicas -en un contexto de frontera entre dos países - tiene sus ventajas. Marco Antonio Samaniego (2006), por ejemplo, sugirió ya el doble carácter de las obras de defensa contra inundaciones en el gran marco de estudio de los ríos internacionales. Por un lado, no solamente fueron necesarias para proteger ciudades y centros de población, sino que las inundaciones periódicas también mantenían en funcionamiento un ecosistema particular en las ciénegas del delta del río Colorado. Asimismo, contener el exceso de agua en crecientes o avenidas, devino en obras de canalización que al mismo tiempo se aprovecharon para desarrollar grandes áreas de cultivo. Pero las obras de defensa también representaban un oneroso sistema, pues eran costosas y demandaban periódicos trabajos de mantenimiento y reparación; y solamente funcionaron hasta la década de 1940, cuando los trabajos de obras para irrigación obtuvieron preponderancia (Samaniego, 2006, pp. 18-29). Sobra destacar el largo ciclo de transformación del paisaje que esto ocasionó.

La historia de las obras hidráulicas en el norte fronterizo de México goza de un cúmulo de estudios bastante nutrido, imposible de citar aquí por su dimensión. No obstante, sin duda fue Roberto Melville (2002) quien describió con más detalle las implicaciones prácticas del doble carácter de dicho tipo de obras, 
las cuales incluso provocaron confrontaciones dentro del Estado, entre los planes diseñados por la Secretaría de Comunicaciones y Obras Públicas (SCOP) y los de la Comisión Nacional de Irrigación (CNI) para una sección del Bajo Río Bravo (pp. 199-200). Pocos años antes de la celebración de un tratado para la repartición equitativa de aguas del Río Bravo del Norte, y frente a las constantes inundaciones por crecidas de aguas que sufrían las poblaciones ribereñas, en el año de 1936 el ingeniero Eduardo Chávez ideó un sistema para aprovechar los excedentes y de paso emprender un proyecto de colonización con una red de irrigación. Se trataba del famoso Tajo del Retamal, que se aprovecharía para establecer repatriados en varias colonias o nuevas poblaciones (Melville, 2002, pp. 204-205). Incluso se publicó un decreto en 1939, dejando a cargo de una "dirección de obras de defensa" todos los trabajos de planificación y ejecución, ${ }^{2}$ aunque los ámbitos de acción entre instancias administrativas fueron sumamente ambiguos, pues no se modificaron las competencias de cada dependencia involucrada, dejando la empresa "al carisma y buena voluntad de los cooperantes" (Melville, 2002, p. 206).

Sin embargo, explicar cuáles sensibilidades y criterios regían las obras de defensa, y cómo se llegó a este punto, requiere descender al plano de la evidencia histórica. En los estudios sobre irrigación la atención en las obras de defensa fue rara y en ocasiones inexistente. Es el caso de los diversos trabajos de Luis Aboites (1987), donde las obras se presentan como un fin y destino definidos, a través de grandes presas, pero sin destacar sus implicaciones colaterales en sitios donde se transitaba desde el modelo de mejoras materiales locales hacia otro de obras públicas nacionales. Algo que destaca en este tipo de estudios es la presencia de dos requisitos para la construcción del Estado: la existencia de una burocracia técnica, por un lado, y la tendencia a centralizar decisiones —como necesidad administrativa de las grandes obras para su ejecución sostenida-, por el otro (Walsh, 2012, pp. 51-52).

2 La oficina a través del tiempo fue nombrada de varias maneras: Comisión de Obras de Defensa contra Inundaciones del Río Bravo, Comisión de Defensa contra el Río Bravo, Dirección de Obras del Río Bravo, entre otras. En adelante se usará Comisión de Obras de Defensa (COD), a menos que los expedientes indiquen otra locución. 
Asoma así la necesidad de entender cómo sucedió el tránsito desde una procuración de obras de carácter local hacia otra de corte centralizado, como lo han hecho algunos estudios de historia de la construcción. Casey Walsh (2008), historiador sensible a las implicaciones de la demarcación de ramos de obras públicas, señaló que, por un lado, el lenguaje del fomento es histórico y programático, pues articula un sentido de movimiento desde el pasado hacia el futuro y postula una determinada condición futura; pero, por otro lado,

...no se presta al negocio cotidiano de la edificación o mantenimiento de bordos para control de inundaciones, organización del crédito, excavación de canales, establecimiento de ejidos, y todas las demás cosas que componen el desarrollo, aunque este lenguaje diario de la burocracia y trabajo puede ayudar a lograr los objetivos a largo plazo del fomento" (Walsh, 2008). ${ }^{3}$

Algunos historiadores de la frontera México-Estados Unidos han identificado hechos con implicaciones técnicas importantes. Marco Antonio Samaniego (2006), por ejemplo, sugirió la posición favorable de México en los tratados de negociación de reparto de aguas, indicando que los intentos por desarrollar las políticas de irrigación respondieron precisamente a esa circunstancia: la necesidad de equiparar las condiciones de la negociación, porque había que demostrar que se estaría en condición de usar el agua reclamada (p. 50). Martín González (2017), en otro ejemplo, dio por descontado que la construcción de obras de defensa implicaba fijar el curso del Río Bravo, sin advertir la lógica del pensamiento técnico (p. 143). En estas investigaciones el técnico y su campo no pasan de ser ilustrativos de las decisiones políticas y los agentes de los mercados económicos. La consecuencia de una acción técnica para nada implica que esa haya sido su intención, pues, siguiendo a Brian Larkin (2013, pp. 328-329), las sucesiones de infraestructuras en el tiempo sugieren la necesidad de distinguir entre los cambios que fueron parte de la evolución de un sistema

3 La traducción es nuestra. 
y aquellos que se introdujeron de manera artificial. Sin embargo, nuevos estudios como el de C. J. Álvarez (2019a) explican cómo las primeras obras de control en la frontera sur de los Estados Unidos estuvieron precisamente dirigidas desde la International Boundary Commission (IBC), ámbito burocrático que de vigilar los primeros trabajos de riego en la frontera pasó a constituirse al paso de las décadas en una verdadera oficina de ingeniería que, literalmente, construyó la frontera cuando erigió su primera valla. Asimismo, Álvarez (2019b) sugiere claramente las ventajas de comprender el espacio de los técnicos, a través de identificar la función que hicieron de mediadores (pp. 233-236). En otro ejemplo, Justin Castro (2019) se refiere a la manera en que el ingeniero Modesto Rolland sorteó cambios de régimen en el México posrevolucionario, a través de sus habilidades retóricas y políticas, consciente de las implicaciones de sus decisiones en el ramo de obras hidráulicas y puertos libres (pp. 122, 177 y ss.).

De cara a todo lo anterior, es importante saber que en el borde territorial del norte, entre los siglos XIX y XX en México, ya se encontraban establecidos grupos de constructores reunidos en comisiones especiales; esto sugiere que la manera en que se construyó el Estado a través de sus obras no fue para nada un asunto sencillo, y mucho menos fue algo unificado en sus criterios. Se abre, de esta forma, un espacio para interrogar el proceso de federalización a través de la técnica. Desde este punto de vista, la descripción de características de las obras puede revestir un nuevo interés, pues su definición no fue resultado de una sola mente o decisión, sino que se resolvió dentro de un campo en disputa. Su ubicación, sus dimensiones y sus materiales y técnicas de construcción constituyeron factores con implicaciones muy específicas en el uso del suelo y de los recursos; los responsables lo sabían y tomaron partido a través de elecciones específicas.

A través de varios ejemplos de obras proyectadas en ciudades fronterizas, en este artículo se ejemplifican las discusiones en torno a los proyectos en ramos de obra pública como la defensa contra inundaciones, la proyectación de edificios federales y en general el mejoramiento material de las localidades. En estos tres 
ámbitos interesa destacar el conflicto entre técnicos de distintas oficinas de la administración pública y sobre todo el desenlace a través de opciones alternativas.

En un primer apartado de este texto se discute el contexto geográfico y temporal de las obras de defensa; en el segundo, se revisan los primeros trabajos que se llevaron a cabo en Matamoros y Ciudad Juárez, destacando cómo los técnicos de obras de defensa tuvieron que ubicarse entre los requerimientos crecientes de la Comisión Internacional de Límites (CIL) y la paulatina definición de este ramo de obras dentro de la Secretaría de Fomento, Colonización, Industria y Comercio (SFCIC) y a partir de 1891 de su sucesora, la SCOP. En las siguientes tres secciones se ofrecen varios casos de estudio relativos a Nogales, Ciudad Juárez y varias localidades del Bajo Río Bravo, con el fin de destacar la escasa demarcación de los campos de acción de los ingenieros y su perfil versátil que tendía a plantear soluciones pragmáticas en el proceso de superación de convulsiones revolucionarias de las décadas de 1910 y 1920. Por último, se ofrece una discusión sobre la relevancia de "bajar a la historia" de la mano de la evidencia documental que destaca la necesidad de más estudios sobre las burocracias técnicas.

\section{LAS OBRAS DE DEFENSA EN LOS CAUCES DE AGUA DE LA FRONTERA}

Entre los años de 1879 y 1944, en las poblaciones y ciudades fronterizas del norte de México, varios grupos de ingenieros del gobierno se ocuparon de proyectar y ejecutar obras de defensa contra inundaciones. El Río Bravo, que marcaba una parte de la línea divisoria con los Estados Unidos de Norteamérica, presentaba crecientes de caudal entre los meses de marzo y mayo hasta el punto de inundar — de forma periódica y recurrente- variedad de comunidades y ciudades. Como resultado de esta dinámica, el río cambiaba sensiblemente de trayecto, abandonando viejos cauces y abriendo otros nuevos de forma a veces brusca o paulatina, lo que provocaba a su vez conflictos entre los habitantes de uno y 
otro lado de la frontera, pues llegaban a separarse fragmentos de tierra de no poca dimensión (los llamados bancos). De igual manera, en los demás sitios de la frontera terrestre se enfrentaban problemas parecidos, con motivo de escurrimientos o cañones naturales que liberaban el exceso de agua por lluvias o derretimiento de nieve en las partes altas. Así, en la línea divisoria de tierra había que plantear los trabajos de uno y otro lado de modo coordinado para ayudar al desalojo de exceso de agua. En estas décadas las ciudades y poblados fronterizos crecieron de modo importante, y la particularidad de las obras de defensa hizo que los técnicos encargados asumieran otras responsabilidades adicionales con relación a la urbanización y las mejoras materiales. Sin embargo, por el otro lado las inundaciones representaban ventajas para los agricultores, principalmente por el entarquinamiento de suelos, pues se beneficiaban de limos y nutrientes que eran arrastrados por las avenidas periódicas de agua. Al paso de los años se fue agregando a este complejo y cambiante contexto una variable más: la definición de criterios para resolver la fijación definitiva de la línea divisoria entre México y Estados Unidos.

Con anterioridad, las localidades fronterizas ya enfrentaban los problemas y las ventajas de las inundaciones en la esfera de sus asuntos locales; sin embargo, con ocasión de los conflictos por segregación o pérdida de territorio debido al cambio de cauce del río, los propietarios afectados promovieron trámites que pasaban por jueces y cónsules, hasta llegar algunas veces a la Secretaría de Relaciones Exteriores (SRE). Lo común era que las poblaciones ribereñas emprendieran obras de canalización y rebalse para el riego, y del mismo modo realizaban trabajos - muy diversos, y periódicos - para protegerse de las inundaciones. Lidiar en el plano local con el movimiento del Río Bravo no fue sencillo, pues por un lado las obras de irrigación —dependiendo de su tamañopodían favorecer que el río cambiara de trayecto, especialmente en los momentos de bruscas crecientes de caudal; y por el otro lado, considerando el curso sinuoso de algunos tramos del río, demasiadas obras de reforzamiento de los bordos de un lado podían incrementar la violencia de empuje del agua sobre la orilla 
contraria acentuando los procesos naturales de erosión y avulsión del suelo. Si bien desde 1848 y 1852, cuando se definió la línea divisoria entre ambos países, ya se había reunido una "comisión de límites”, sus trabajos apenas abarcaron la elaboración de la cartografía del Río Bravo y el área de la frontera terrestre, además de la construcción de algunos monumentos para señalar la línea. De tal manera, los municipios debieron lidiar con los problemas y oportunidades que representaban las inundaciones, y emprender trabajos que necesariamente requerían gastos importantes. Desde 1865, por ejemplo, en el área de Matamoros los vecinos, a pesar de las protestas de los norteamericanos, construyeron una estacada o "jardín flotante" que al tiempo que servía como protección de la ribera también era usado como lugar de recreo; poco tiempo después una creciente derribó la estructura por completo. Lo mismo sucedió del lado norteamericano del río, cuando el señor Belden construyó un malecón, también denominado jetty, con las subsiguientes protestas de mexicanos (SRE, 1903, pp. 14-15).

Los trabajos que se emprendieron en estas circunstancias merecen una descripción, pues su concepción técnica y el modo en que se organizaron y financiaron permiten discutir los mecanismos a los que recurrían los municipios para asegurar las mejoras materiales sin grandes recursos. Por ejemplo, en la villa Paso del Norte -después renombrada como Ciudad Juárez- los trabajos consistían en el reforzamiento de las riberas mediante plantación de árboles y terraplenados con fajinas de ramas, y en la construcción de presas temporales también llamadas rebalses; estos se organizaban cada año de forma periódica y se distribuían fatigas entre los vecinos, igualmente se recibían donaciones de material. Los primeros trabajos consistían en consolidar tramos de la orilla y se ejecutaron meses antes de la llegada de la primavera, mientras que los segundos tenían como finalidad aprovechar al máximo el caudal de agua con fines de irrigación. Las crecientes repentinas por supuesto que marcaban el carácter temporal y efímero de estas obras, que cada año había que volver a reconstruir y reforzar. Los gastos llegaron a ser tan fuertes y constantes, que el ingeniero Agustín Barbachano — con la idea de liberar al ayuntamiento 
de estos gastos periódicos - propuso en 1872 que se organizara una compañía de obras, una “compañía filantrópica-mercantil” que estuviera financiada por los principales propietarios de la villa. ${ }^{4}$ Incluso varias décadas después, a pesar del arribo de los técnicos del gobierno mexicano, continuaron empleándose métodos tradicionales debido a que eran intervenciones pasivas y tendían a modificar en leve medida el paisaje ribereño.

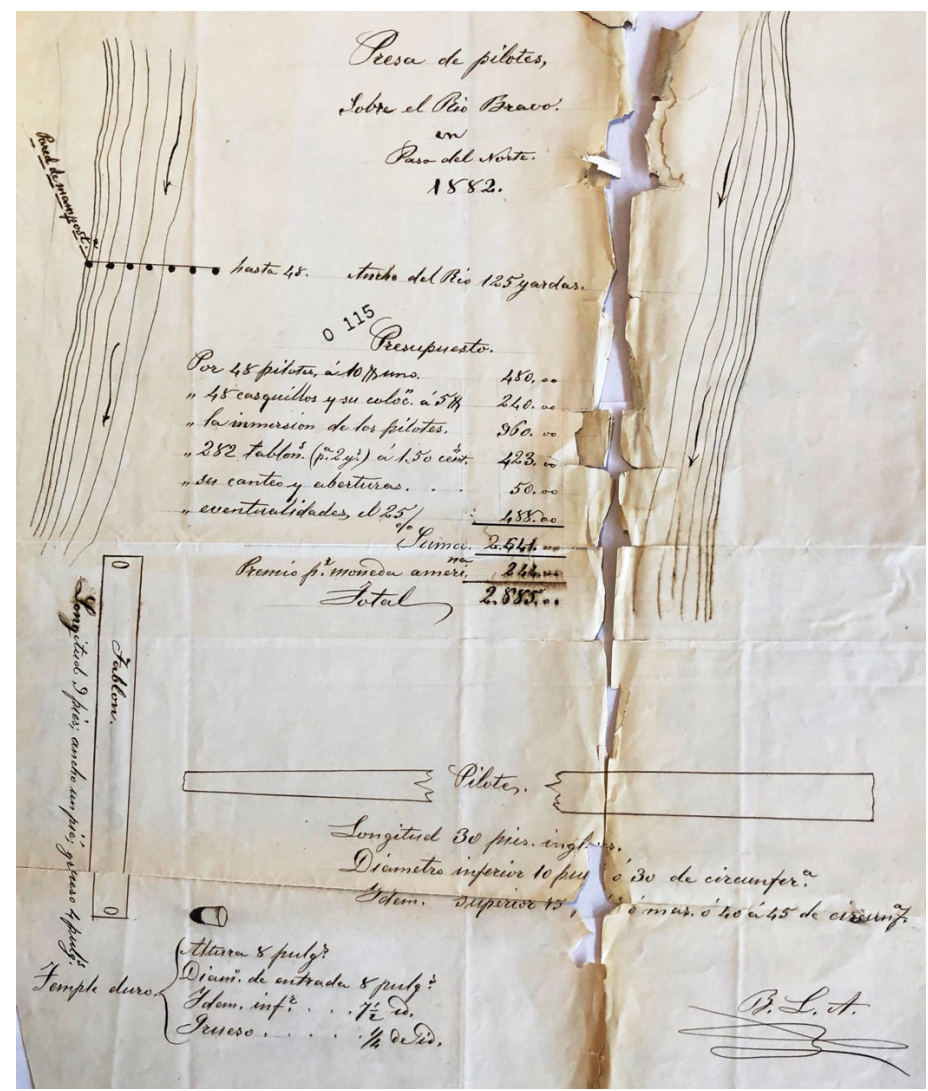

Figura 1. Estas obras eran provisionales, pues había que reconstruirlas cada año. Ejemplo de un proyecto para presa (rebalse) de pilotes y tablones, por el ingeniero Benito León Acosta, 1882 (AHCJ, PyT, Gob, Caja 18, Libro 293).

4 AHHP, FSXIX, Caja 389, Exp. 1. 
Solamente hasta el arribo de las vías de varias compañías ferroviarias -entre las décadas de 1880 y 1890- puede decirse que inició el crecimiento sostenido de poblaciones como Nogales, Ciudad Juárez, Piedras Negras y Nuevo Laredo. Matamoros, aunque no viera llegar el ferrocarril hasta el año de 1905, fue un caso especial por su estatuto de puerto, si bien se hallaba alejado cinco kilómetros de la bahía y en un área donde el Río Bravo fluía en curvas muy cerradas antes de desembocar en el Golfo de México. La necesidad de proteger estas y otras localidades cobró importancia, tanto el incremento de flujo y cruces de mercancía en ferrocarril constituyeron factores para que el gobierno mexicano comenzara a ejercer una mayor vigilancia. Tal vez las decisiones más relevantes en este contexto fueron dos: el arribo en 1879 a Matamoros de un ingeniero del ramo "Obras en puertos", dependiente de la SFCIC, y la dotación de estructura burocrática a la CIL en los inicios de la década de 1890.

La construcción de ferrocarriles tuvo dos consecuencias negativas en la problemática de las inundaciones. Por un lado, las plataformas sobre las cuales se desplantaron las vías constituían verdaderos diques dentro de las redes locales de irrigación y aunque contaron con sus desagües, estos no siempre fueron suficientes o funcionaron. Por otro lado, los puentes construidos implicaron riesgos de atasco del material que era arrastrado con las avenidas, incrementando el peligro de derribo de dichas estructuras o la multiplicación de la fuerza del agua sobre los bordos de las orillas. Incluso sucedió que se construyeron plataformas de vías paralelas muy próximas a la ribera del Río Bravo, que en términos prácticos constituyeron una obra de defensa o reforzamiento de la ribera; este fue el caso de una investigación con motivo de la segregación de un banco que al paso del tiempo cobraría fama: el Chamizal (SRE, 1903, pp. 42-63).

La CIL se fortaleció a partir de la atención de problemas debidos al cambio de posición del río y más adelante también se involucró en la vigilancia de obras de defensa, la promoción de obras de enderezamiento del río y, sobre todo, la gestión de trata- 
dos para la distribución equitativa del agua de los ríos entre ambos países. Todas estas acciones tuvieron muchas implicaciones técnicas importantes. Así, hasta la década de 1910 en Matamoros y Ciudad Juárez se llevaron a cabo obras de defensa intermitentes y sin una visión a largo plazo, solamente hasta la década de 1930 iniciaron trabajos en otras ciudades como Nuevo Laredo, Piedras Negras y Nogales, e incluso en localidades más pequeñas que cumplían funciones de cruce entre ambos países. La particularidad de estas obras es que se concibieron como programas permanentes de trabajos. La relación con la CIL, puede comprenderse con facilidad, resultó compleja, pues en un primer periodo — cuando menos hasta la década de 1920- el ingeniero consultor dependía de la SFCIC y no fue raro ver opiniones encontradas respecto a lo que se aconsejaba hacer desde el punto de vista técnico y lo que, por el contrario, se planteaba desde el campo diplomático (Bustamante, 1999, p. 65). Se trata de un rubro que merece más estudios, ya que por lo general los investigadores acuden a fondos documentales distintos para abordar los mismos problemas. El primer comisionado, el general Pedro García Conde, fue perito facultativo de minas y aunque después hubo varios licenciados, destacaron a través del tiempo los ingenieros. También falta esclarecer sus encargos paralelos en varias oficinas de gobierno, por ejemplo, Fernando Beltrán y Puga, a decir de su expediente personal, durante el tiempo en que fungió como comisionado mexicano de la CIL, en el periodo de 1904-1924, también fue nombrado por la SFCIC como inspector del río Colorado en 1912 (International Boundary and Water Comission [IBWC], 1938). ${ }^{5}$

\section{LAS OBRAS DE DEFENSA EN EL ESPACIO ENTRE COMISIONES}

Sería largo y complejo intentar un recuento de cómo fue haciéndose efectiva la presencia del gobierno mexicano en los territorios del norte de México, desde inicios del siglo XIX en adelante. Pero sin duda podría hablarse de la organización de expediciones e

5 AHSRE, LE-1329. 
inspecciones que perseguían objetivos variados, acompañadas de un paulatino establecimiento de oficinas del gobierno central o federal.

Después de la guerra con Estados Unidos se acordó en el plano diplomático la creación de una línea divisoria en 1848 y se firmó el Tratado de Paz, Amistad y Límites y Arreglo Definitivo entre la República Mexicana y los Estados Unidos de América, también llamado Tratado de Guadalupe Hidalgo. ${ }^{6}$ Respecto a la línea divisoria, su artículo V indicaba que tomando el Río Bravo, o Río Grande, como línea divisoria, debería seguirse siempre el canal más profundo en caso de que hubiera varios brazos (SRE, 1896, p. 1). Casi inmediatamente se formó una comisión, donde figuraron el general Pedro García Conde en calidad de comisario y el agrimensor José Salazar Ilárregui, acompañados de ayudantes y una escolta; luego se constituiría una equivalente del lado de la Unión Americana, con fines de reconocimiento y demarcación de la línea (SRE, 1896, p. 2). En sus primeros años de funcionamiento la SFCIC informaba que ya se hallaban concluidos los planos para unas obras que se llevarían a cabo en el puerto de Matamoros, dentro del ramo "Aduanas y almacenes en los puertos" (SFCIC, 1857, p. 29). ${ }^{7}$

Dos protagonistas de las obras de defensa fueron Ignacio Garfias y Felipe Zavalza; en 1858 habían decidido salirse del Colegio de Minería -después de un periodo de disturbios y protestas estudiantiles - para ingresar a la Academia de Bellas Artes de San Carlos y concluir con sus estudios (Álvarez, 1906, p. 25). Probablemente el nuevo plan de la carrera de ingeniero-arquitecto les había parecido demasiado largo, pero años después, ya en Paso del Norte, llegó a ser muy evidente la ventaja de sus amplios conocimientos, ambos habían hecho trabajos de ingeniería y arquitectura. Durante la década de 1870 Garfias estuvo a cargo de elaborar un informe para la SFCIC sobre inundaciones en el Valle de México; en su brevísimo escrito, parece evidente que estaba al tanto de las discusiones sobre el mejor método a seguir para des-

6 Pocos años después se reformuló, en el denominado Tratado de la Mesilla.

7 No hemos podido localizar dichos planos. 
alojar las aguas y de los proyectos de Ricardo de Garay y Ricardo Orozco (SFCIC, 1877, pp. 367-368). Quizás por este antecedente, la SFCIC y la SRE, en el año de 1877, encomendaron a Garfias una inspección de los trabajos de reforzamiento que se habían ejecutado en la ribera norteamericana del Río Bravo, debido a que dichas obras podían tener consecuencias negativas en la parte mexicana. Garfias no solamente revisó un caso específico en Fort Brown, frente a Matamoros, sino que también emprendió estudios sobre el comportamiento del afluente y razones del cambio de cauce, elaboró planos topográficos y dibujos de cortes transversales del río, y también organizó y ejecutó los trabajos de reforzamiento de la ribera mexicana (SFCIC, 1885, tomo 3, pp. 47, 49). ${ }^{8}$

En las áreas adyacentes a la línea fronteriza venían estableciéndose poblaciones, de esa manera, con el movimiento del Río Bravo -y el denuncio de minas-, comenzaron a surgir problemas acerca de las posiciones exactas de los monumentos y la línea divisoria. Llegaron quejas hasta las oficinas de la SRE y así ambos gobiernos decidieron celebrar una nueva convención que se firmó en julio de 1882, con el objetivo de revisar cuáles de los viejos monumentos todavía persistían en sus sitios y discutir y decidir cuántos monumentos más había que levantar, pues hasta el momento solamente existían 53 del lado mexicano y 46 del norteamericano (SRE, s.f.). ${ }^{9}$ De tal modo, se formó una comisión de reconocimiento con varios ingenieros, incluido un astrónomo práctico; lo mismo hicieron los norteamericanos, y cuando ambas se hallaban reunidas constituían la Comisión Internacional de Límites (SRE, 1896). En el terreno práctico, aunque se construyeron algunos nuevos monumentos, no se alcanzaron las metas planteadas, por lo que se celebró otra convención semejante en 1884 para intentar concluir los trabajos (SRE, s.f.). ${ }^{10}$

8 Ignació Garfias arribó a Matamoros en agosto de 1879 y concluyó los trabajos en julio de 1882.

9 La "Convención para reponer monumentos que marcan la línea divisoria entre Paso del Norte y el Océano Pacífico", acordada en 1882 y ratificada hasta 1883, también se conoció como Tratado de Remonumentación.

10 La "Convención respecto a la línea divisoria entre los dos países en la parte que sigue el lecho del Río Grande y del Río Gila”, acordada 1884 y ratificada hasta 1886, también se conoció como Tratado de la Línea Fija. 
Los informes de la SFCIC revelaban la atención dispersa de la frontera. En el rubro de "Obras en puertos", por ejemplo, se referían las obras hidráulicas que se habían comenzado en el puerto de Matamoros a cargo de Ignacio Garfias (SFCIC, 1885, tomo 3, pp. 47-58); mientras que en el ramo "Líneas de navegación" se abordaban los asuntos relativos a la línea divisoria entre México y Estados Unidos, con informes de una sección militar de reconocimiento de 1882 que ascendió a comisión científica el año siguiente (SFCIC, 1885, tomo 1, pp. 32-55). En el rubro de "Puentes" se incluían los contratos para los puentes de cruce internacional en 1884 (SFCIC, 1885, tomo 2, p. 244); además, el apartado "Canales de navegación, ríos y lagos" describía las obras de defensa a llevarse a cabo en Paso del Norte por el ingeniero Felipe Zavalza (SFCIC, 1885, tomo 3, p. 258).

Desde el punto de vista científico, los trabajos e informes de Garfias tuvieron repercusiones importantes, pues pocos años después fue citado en arbitrajes internacionales por los ingenieros de la CIL, con motivo del nuevo capítulo de actividad que inició desde $1882 .{ }^{11}$ Un evento que había desencadenado esta nueva convención, fue la segregación de la Isla de Morteritos - una modalidad de banco- y su invasión por norteamericanos. Además de ubicar los monumentos que indicaban la línea internacional, los ingenieros de la CIL abordaron el problema de los "bancos", y el estudio de Garfias constituyó un precedente de importancia. La observación del comportamiento del cauce durante periodos de crecientes y de escasez de agua daba como resultado fenómenos diversos de desgaste en las partes convexa y cóncava de las riberas, esto — dada la constitución estratigráfica del suelo- podía dar lugar a procesos de erosión y avulsión (SRE, 1903, pp. 11 y ss.).

Desde la perspectiva práctica, los trabajos de reforzamiento que dirigió Garfias se ejecutaron sin ningún antecedente; aunque tenían presentes los grandes y ambiciosos trabajos que se venían construyendo sobre el río Misisipi, Garfias comentó que las obras

11 AHSRE, LE-1161. Otro nombre que en el plano burocrático tuvo esta comisión, durante el año de 1883, fue Comisión Internacional de Límites Fluviales. 
mexicanas serían más modestas. Consistían básicamente en enrocamientos colocados entre armazones de madera previamente incrustados en el subsuelo de la ribera, todo sujetado entre sí por alambres (SFCIC, 1885, tomo 3, pp. 51-54).

En los siguientes años se definieron los nombramientos de la Sección Militar de Reconocimiento y de la Comisión Científica (SFCIC, 1887, tomo 1, pp. 32-34). Se elaboraron informes sobre los sitios donde se ubicaban los monumentos que había construido la CIL treinta años atrás, algunos fueron imposibles de localizar. También se daban recomendaciones diversas, como promover el asentamiento de poblaciones en la cercanía de dichos monumentos, pero principalmente se hicieron mediciones para corregir la cartografía disponible (SFCIC, 1887, tomo 1, pp. 35-55). Algo importante que iría aflorando en estos trabajos — casi paralelos a los de la CIL- fue la necesidad de modificar algunos artículos del Tratado de Guadalupe Hidalgo, respecto a los movimientos del río, cambios de cauce y formación de bancos, estas modificaciones se concretaron más adelante con la firma de un nuevo documento.

La SFCIC en 1887 presentó un informe del jefe de la Sección de Cartografía, J. Molina, firmado en 1885, donde se indicaban dos planos (calcas) que abarcaban desde Paso del Norte, hasta el extremo occidental de la línea fronteriza, es decir, hacia el océano Pacífico; se destinarían para auxiliar el trabajo de técnicos de "las comisiones exploradoras y de rectificación de límites" (SFCIC, 1887, tomo 5). Sin embargo, continuaba aflorando la dispersión de asuntos, pues en la sección de "Contratos" se hablaba de uno que se celebraría con Antonio Canales para construir varios puentes en el Río Bravo (SFCIC, 1887, tomo 2, pp. 244-245) y en la sección de "Obras en puertos" se daban muy breves descripciones de los trabajos de Garfias, pues se ocuparon más en los fondeaderos de la bahía (SFCIC, 1887, tomo 3, pp. 2-30) y en la comisión de Felipe Zavalza, presentada como Inspección del Ferrocarril Central Mexicano, se incluyó un presupuesto de obras de defensa, pues las plataformas de las vías tenían efectos perniciosos en el Barrio del Chamizal (SFCIC, 1887, tomo 3, p. 258). 
La presencia de Garfias en la frontera antecedió a la firma de otra convención que estableció de modo definitivo la CIL, dotándola de una estructura burocrática definida en el año de 1891 (SRE, s. f.). ${ }^{12}$ A partir de entonces, el ingeniero consultor (o ingeniero en jefe) dependería de la SFCIC, así como también los dos astrónomos adjuntos. Del lado norteamericano se formó también un grupo de técnicos, aunque dependieron todos del Departamento del Interior. El acto de formalización de la CIL —entre ambos países - se celebró en la aduana de Ciudad Juárez, en Noviembre de 1891 (SRE, 1896). Llama la atención que el mismo año, una parte no menor de los quehaceres de la SFCIC se trasladó a la recién creada SCOP, donde en adelante quedaría adscrita la oficina de obras de defensa en ciernes.

Los informes de la CIL en este periodo resultan interesantes porque adquirieron carácter sistemático. Los trabajos consistían en inspeccionar todos los monumentos y sustituir los desaparecidos o dañados, y construir nuevos. En las áreas de ríos, como el caso del río Tijuana, una creciente había hecho desaparecer por completo el monumento de granito número 255. Las mediciones astronómicas para revisar la fijación de la línea divisoria se hacían de noche, estableciendo determinaciones a partir de las estrellas y empleando señales telegráficas; en este caso incluso se estudiaron los diarios y libretas originales que Salazar y Emory habían elaborado en la década de 1850. Algunas medidas que debían practicarse en medio de los desiertos tuvieron que posponerse, pues no había condiciones para trabajar y ni siquiera se disponía de agua potable (SRE, 1896). Asimismo, se sumaron otras circunstancias, pues por segunda vez se involucró a la oficina de obras de defensa en la producción de datos que requería la CIL para resolver cómo había que proceder ante el problema de los bancos. Garfias no se alejó de Ciudad Juárez, pues en el año de 1897 elaboró —con los ingenieros G. Montiel, Antonio García Cubas y José Covarrubiasel Informe sobre la formación de bancos para la CIL. El motivo de

12 La "Convención para el establecimiento de una Comisión Internacional de Límites, que decida las cuestiones que se susciten en el cauce de los ríos Bravo del Norte y Colorado" se firmó en 1889 y se ratificó hasta 1891. 
este estudio fue la necesidad largamente postergada de reconsiderar dos artículos del Tratado de la Línea Fija de 1884 para que en adelante se enfrentara de manera más práctica la segregación de bancos debido a los cambios de cauce del Río Bravo. ${ }^{13}$

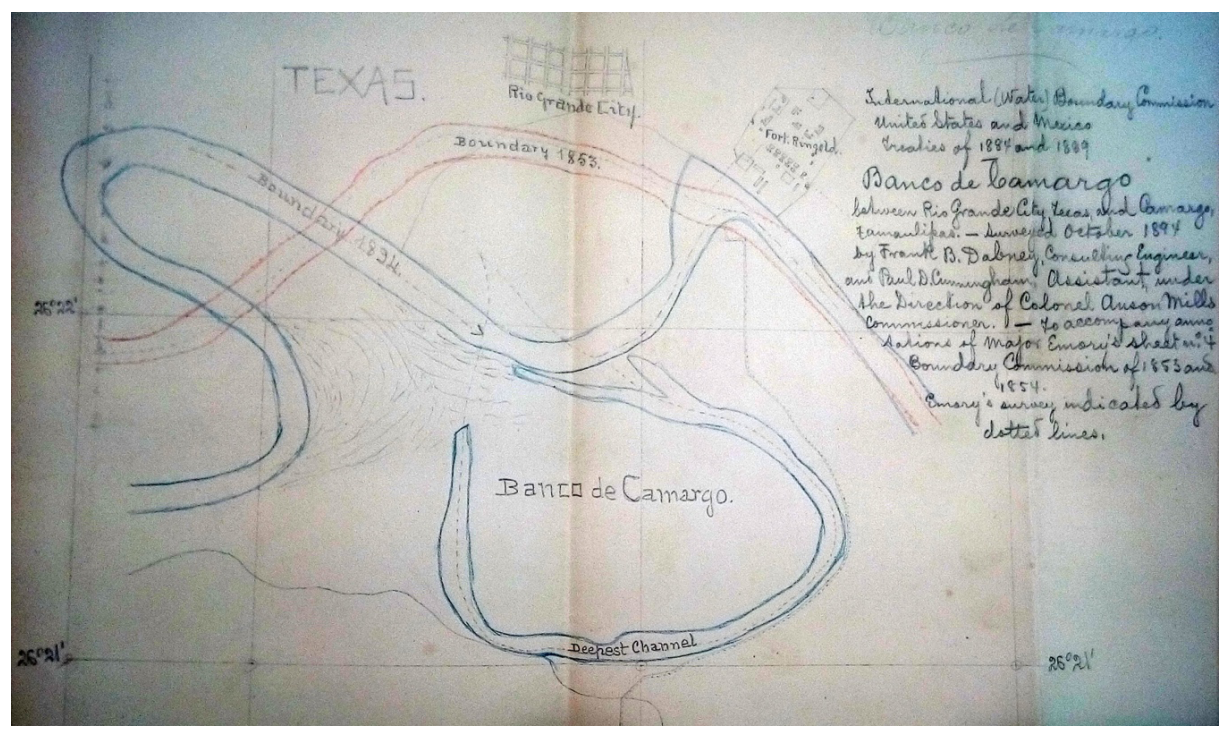

Figura 2. Los ingenieros de las obras de defensa recorrieron y llegaron a conocer con detalle muchos tramos de la frontera fluvial. Estudio del movimiento del Río Bravo y la conformación del banco Camargo, por Manuel

María Mendiola, 1896 (AGN, LEUA, Caja 4, Legajo 1-A, Exp. 27).

Otro ingeniero de la primera etapa de obras fue Manuel María Mendiola, ${ }^{14}$ quien llegó desde Matamoros a Ciudad Juárez con el encargo de revisar las obras de defensa de la ciudad; ya traía consigo el puesto de jefe de las obras de defensa en ese puerto del golfo desde $1903 .{ }^{15}$ Los diversos informes de la CIL, por otra

13 AHCILA, Gaveta 3, Exp. 1214.

14 Los datos sobre su formación profesional son vagos, a veces incluso resultan contradictorios. En una ocasión informó que había obtenido su título de ingeniero en el Instituto Juan José de la Garza, establecido en Matamoros; pero en otro momento indicó que lo había recibido del Estado Mayor del Ejército. Véase AHCJ, PyT, Caja 28, Exp. 1.

15 AHCJ, PyT, Caja 86, Exp. 1. 
parte, indican que cuando menos desde 1894 Mendiola ya venía elaborando estudios y planos, pues dichos trabajos "del capitán Mendiola” eran frecuentemente citados (SRE, 1903, p. 22). Es difícil conocer los motivos de esta asignación en Ciudad Juárez, pero coincidió con un momento en que se estaba definiendo el plano de una ciudad moderna que regularía el crecimiento en el futuro, con ello también dio inicio una nueva etapa de regulaciones en construcción. Dichos trabajos antecedentes pueden apreciarse con mucho detalle en uno de los estudios que Mendiola presentó para la CIL, aunque comisionado por la SCOP. Su Informe sobre la formación de bancos, fechado en 1896, orientaría en el futuro algunas decisiones, pues había efectuado una considerable investigación documental y trabajos de inspección en terreno. Tomando como base el informe elaborado por el general Cushing en un dictamen del año de 1856 y otros datos, planteó que el Río Bravo, entre Ciudad Juárez y Matamoros, podía dividirse en tres tipos de tramos. En el primero, entre Ciudad Juárez y Presidio del Norte, el río presentaba una fuerte pendiente de recorrido, y dada la consistencia firme de los suelos el río tendía a cambiar de cauce y formar pocos bancos pero de longitud considerable; el segundo tramo, entre Presidio del Norte y Río Grande City se caracterizaba por una pendiente mucho mayor que la primera, con riberas sólidas y resistentes - por estar formadas muchas veces de roca- y por consiguiente con pocos cambios en el canal. El tercer tramo, por el contrario, era extremadamente complejo, pues a lo largo de 108 millas el río recorría terrenos bajos de aluvión, en tierra con poca consistencia, y tan suave pendiente, que el trayecto presentaba sinuosidades sobresalientes, segregando porciones de tierra muy grandes de uno y otro lado. ${ }^{16}$ Los estudios de Mendiola no fueron sencillos, a menudo tuvo que trabajar en lancha, obteniendo muestras dispersas en superficies muy grandes que algunas veces se hallaban inundadas, o en verdaderos pantanos. Decidió estudiar con detenimiento cuatro bancos que, en su opinión, servían de ejemplo para todos los casos de cambios a lo largo del Río Bravo, tomando como criterio los conflictos que

16 AGN, LEUA, Caja 4, Legajo 1-A, Exp. 27. 
se habían presentado entre propietarios de uno y otro lado del río; y elaboró varios apuntes, sobre todo mapas que explicaban el comportamiento del río y aparición de los bancos. La colocación de monumentos frente a estos movimientos resultaba imposible en términos prácticos. ${ }^{17}$ Estos estudios tuvieron una trascendencia muy importante, pues dieron paso a otro tratado que se firmó en el año de 1905 (SRE, s.f.). ${ }^{18}$ En 1909, radicando en Ciudad Juárez, Mendiola también elaboró otro Informe sobre la conformación de bancos para la CIL, con explicaciones de las diversas etapas de su conformación, indicación de los riesgos de obras de irrigación cercanas al río y varias propuestas para contener o conducir los cambios de cauce del río. ${ }^{19}$

A partir de la década de 1920 las obras de defensa contra inundaciones adquirieron una mayor sofisticación, debido a que se incrementó la planta de ingenieros de la Comisión de Obras de Defensa (COD) y los recursos monetarios destinados a los trabajos. También se conformó una sección para operar específicamente en los estados de Coahuila, Nuevo León y Tamaulipas, con sede en Matamoros; el resto del Río Bravo y la frontera terrestre continuarían atendiéndose desde Ciudad Juárez. Mientras las oficinas de Matamoros y Nuevo Laredo quedaron a cargo el ingeniero Luis Pérez Michaud y afrontaron lo que quizás era la sección más compleja del río por la cantidad de afluentes que recibía y el curso tan accidentado del cauce, la de Ciudad Juárez se destinó al cuidado del ingeniero Salvador Arroyo, quien algunas veces tuvo que atender necesidades surgidas en lugares más distantes como Nogales (Sonora). Durante las décadas de 1920 y 1930, los ingenieros de la COD continuaron reflexionando sobre los tipos de obra que debían hacerse con motivo de los mismos problemas, sin embargo, hubo dos diferencias importantes con relación a las épocas anteriores: comenzó a considerarse la rectificación del cauce del Río Bravo y la construcción de canales para el desalojo

17 AGN, LEUA, Caja 4, Legajo 1-A, Exp. 27.

18 La "Convención para evitar las dificultades originadas por los frecuentes cambios a que en su cauce están sujetos los ríos Bravo y Colorado", firmada en 1906 y ratificada en 1907, también se conoció como Tratado de Eliminación de Bancos.

19 AHCILA, Gaveta 3, Exp. 1410. 
de aguas de inundación -lo cual implicó obras de excavación de alguna escala-; también se ejecutaron actividades intensivas de dragado para limpiar los fondos de los cauces y, por otra parte, se recurrió a trabajos de reforzamiento de las riberas más sofisticados y de mayor solidez o permanencia, como las obras de acolchonado del bordo ideadas por el ingeniero Luis Pérez Michaud. ${ }^{20}$

\section{OBRAS DE DEFENSA EN EL DESIERTO Y NOGALES}

El sitio donde se estableció Nogales era un rancho frecuentado por viajeros, en la década de 1850 los técnicos de la CIL construyeron aquí un monumento para indicar la línea divisoria entre ambos países. El incremento del movimiento comercial y el arribo de las vías del ferrocarril en 1882 provocaron cierto auge; el trazado de la población parece que fue elaborado por ingenieros de la compañía de ferrocarril y probablemente se ejecutó de este mismo modo en ambos lados de la frontera (Tinker, 2001; SRE, 1901). ${ }^{21}$ Sin embargo, ambas poblaciones compartían numerosas cañadas que ocasionalmente recibían caudales de agua de no poca dimensión.

Al iniciar el año de 1930 el ingeniero José R. Argüelles arribó a Nogales comisionado por la SCOP para elaborar un diagnóstico de la condición de las oficinas del gobierno federal. Se había trasladado a este punto desde Ciudad Juárez, donde ocupaba el cargo de ingeniero de la recién creada Comisión Nacional de Caminos. Su Informe sobre el agrupamiento de las oficinas federales de Nogales incluyo la elaboración de un programa y un proyecto arquitectónico, y un examen de la topografía del lugar. Según Argüelles, era urgente emprender un estudio de planificación y adaptar la distribución de construcciones a los accidentes del terreno, antes de que hubiera daños mayores, se refería con esto último al peligro de las inundaciones. ${ }^{22}$

20 AGN, CyOP, RyC, Caja 293, Exp. 256.

21 El plano más antiguo de que se tiene noticia data de 1894 y fue elaborado por el ingeniero José González Moreno.

22 AGN, CyOP, DE, Caja 67, Exp. 189786. 


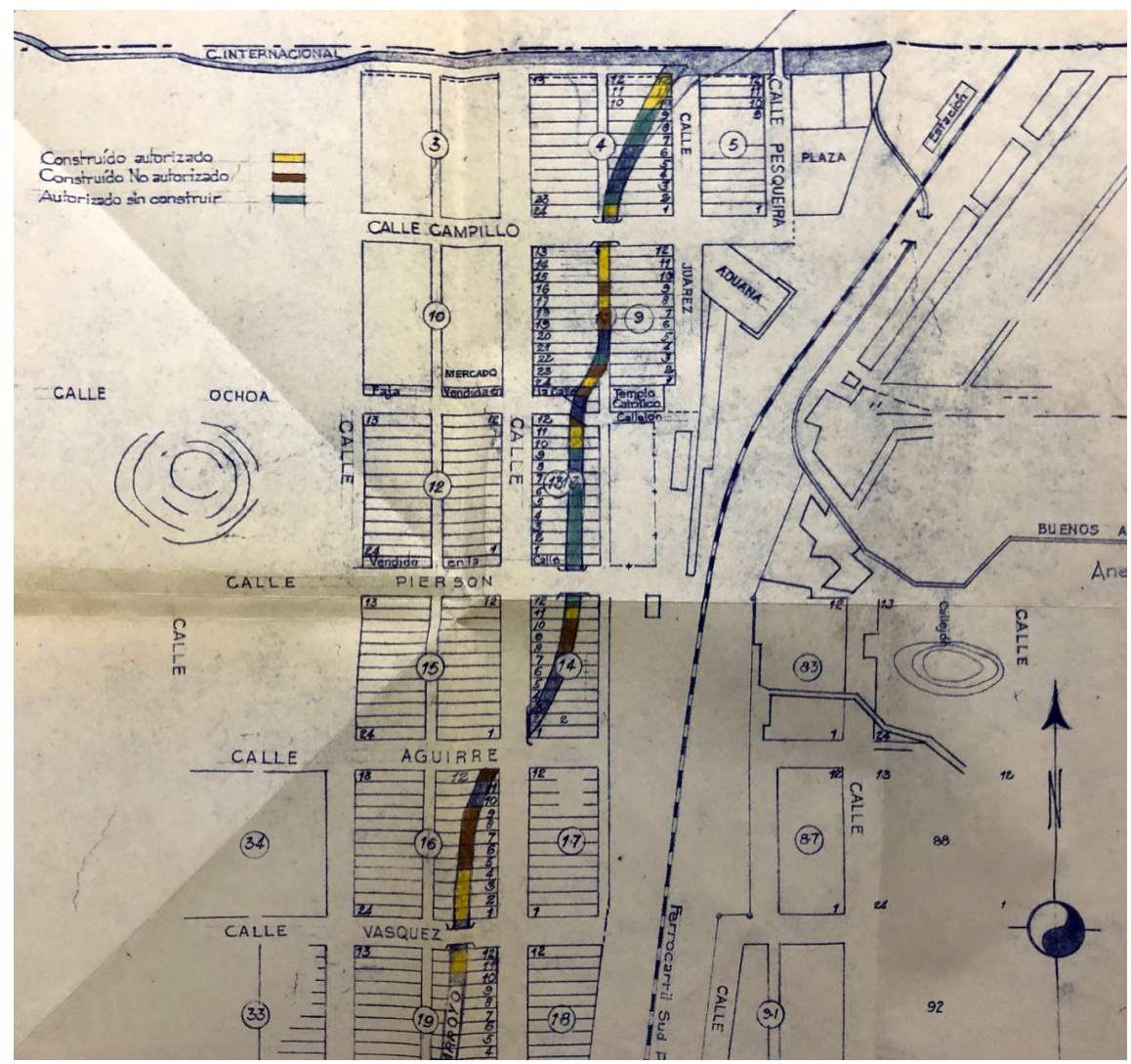

Figura 3. El primer criterio que exigió pensar en un orden para la ciudad se originó en las inundaciones. Registro de los antiguos trabajos de liberación y ocupación del cauce, elaborado por H. M. A. en 1950 (AHCILA, Gaveta 10, Exp. 1096).

Hasta entonces el jefe de la COD, instalado en Ciudad Juárez, hacía visitas para atender el problema de las inundaciones y elaboraba pequeños expedientes que aprobaban obras muy diversas que tendían a cubrir o aproximarse al canal de Nogales. ${ }^{23}$ Pero la inundación en 1930 tuvo resultados tan desastrosos que se promovió la elaboración de informes, como el que firmó el comisionado norteamericano de la IBC, L. W. Lawson, en 1931. La parte mexicana en Nogales era más alta que la ubicada en Arizona; sin

23 AHCILA, Gaveta 10, Exp. 1096. 
embargo, ambas ciudades estaban cruzadas por muchos cañones pequeños que se alimentaban de escurrimientos ocasionales de agua, provenientes de partes altas. Un registro de inundaciones periódicas indicó su ocurrencia en 1905, 1909, 1914, 1926, 1930 y 1931; especialmente la de 1930 había tenido quizás los efectos más desastrosos en pérdida de vidas humanas y daños a propiedades (IBC, 1932, pp. iii-iv). Al parecer ambas municipalidades no habían emprendido ningún trabajo, en busca quizás de apoyos de parte de sus respectivos gobiernos federales, por lo que se acordó que se trataba de un problema de índole internacional y se elaboró un presupuesto por 339 mil dólares que abarcaba no solamente la construcción de dos pequeñas presas, sino también nuevas canalizaciones al interior de las ciudades (IBC, 1932, pp. 2-5). Una implicación de estas obras, además de definir un canal de desalojo en la ciudad mexicana de Nogales, era el ordenamiento de la propiedad porque en muchos casos los edificios se hallaban en cercanía de las áreas de escurrimiento. Mientras un primer tramo del canal corría de forma paralela a las vías del ferrocarril, a partir de cierto punto se introducía al área poblada, pasando por debajo de muchos inmuebles, de modo que se proponía extender el canal cerca de las vías. Nogales, en la parte mexicana, si bien tenía más población que la vecina de Arizona, apenas alcanzaba los 15 mil habitantes y probablemente - salvo el primer trazado que se le dio- no tenía un plan de cómo debía crecer en el futuro ni una autoridad local que regulara las actividades de construcción. En ese tiempo el jefe de las obras de defensa en Nogales fue el ingeniero Alberto Barnetche, de la proyectación y ejecución se encargó Francisco J. Ramírez, de modo que los trabajos se realizaron entre 1934 y 1935 (SCOP, 1936, p. 361) $)^{24}$ y para febrero de 1937 se entregaron las obras al Municipio en una ceremonia pública (SCOP, 1937, p. 148). ${ }^{25}$

Los informes y trabajos de Ramírez describen el contexto en que se concebían los proyectos y se ejecutaban las obras con

24 AHCILA, Gaveta 32, Exp. 1893.

25 AHCILA, Gaveta 32, Exp. 1832. La memoria de la SCOP indicaba que dichas obras -por un monto de 125 mil pesos - se habían concluido solamente en una parte, pues faltaba desarrollar elementos complementarios. 
anterioridad. Existían trabajos que venían realizando técnicos de otras dependencias, que provocaban que hubiera ciertos paralelismos. En el caso de las cañadas más alejadas de la ciudad, aunque conectadas con el canal, un inspector forestal ya había construido pequeñas presas para aminorar el caudal que llegaba a las partes bajas, como sucedió en la cañada de los Héroes. ${ }^{26}$ Pero también dicho inspector había empleado técnicas de arborización para reforzar algunos taludes que podían deslavarse y obstruir el canal; esta fue la misma técnica que Ramírez sugirió emplear: clavar palos de árboles verdes para que con el paso del tiempo arraigaran en el suelo. ${ }^{27}$

Todavía en la década de 1940 continuaron haciéndose más obras. Dado que el canal se había construido con mampostería, nuevas crecientes e inundaciones demostraron que existía el inconveniente del desgaste rápido de superficies, debido al arrastre de materiales gruesos que traía el agua, por lo que ahora habría que revestirlo con cemento armado (SCOP, 1943, p. 78). ${ }^{28}$ Además, se hicieron trabajos para completar todo el sistema, como encauzar el arroyo del Cementerio y levantar rampas y puentes para cruces de automóviles (SCOP, 1944, p. 86). Con motivo de una primera inspección, los ingenieros de la CIL hicieron un recorrido para examinar el estado del canal y hallaron partes invadidas por lo que se recomendaron demoliciones. No parece que el Municipio tuviera capacidad para controlar el crecimiento y las obras de construcción en la ciudad, pues un grupo de diez vecinos envió una solicitud de intervención al ingeniero de la CIL en 1943, para que intercediera ante las autoridades locales debido que, como un rico propietario planteaba, unas obras tendían a obstruir el canal. ${ }^{29}$

A partir del año de 1945 cesarían los trabajos de defensa, pero comenzó a elaborarse un plano regulador, dada la urgencia de

26 AHCILA, Gaveta 32, Exp. 1832. Dicho inspector forestal continuó dependiendo de la Secretaría de Fomento, después de la segregación de la SCOP.

27 AHCILA, Gaveta 32, Exp. 1832.

28 La elección de mampostería también sucedió en otras partes, lo cual sugiere decisiones que en términos de movimiento local de gente y materiales tendían a ser más tradicionales, como se puede observar en muchos otros casos.

29 AHCILA, Gaveta 32, Exp. 1834. 


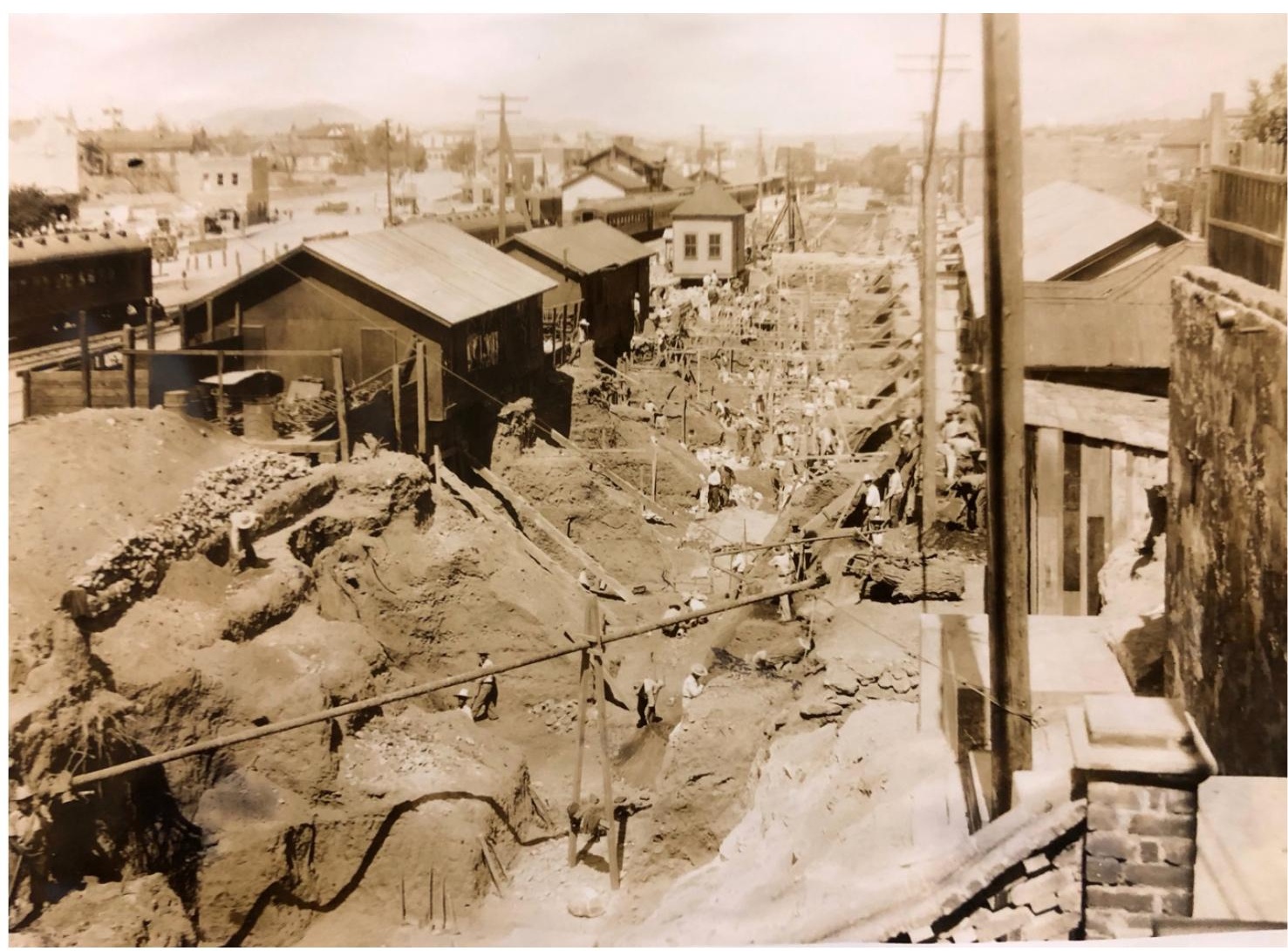

Figura 4. Primeros trabajos de canalización, dirigidos por el ingeniero Francisco J. Ramírez, ca. 1930 (AHCILA, Gaveta 32, Exp. 1834).

ordenar la forma de ocupación de suelo. Solamente hasta la década de 1960 se adoptaría uno, en el periodo en que al frente de la SCOP ya se hallaba el arquitecto Carlos Lazo Barreiro.

\section{CIUDAD JUÁREZ, SEDE DE LAS OBRAS DE DEFENSA}

Ciudad Juárez había sido una de las dos sedes de los ingenieros de obras de defensa ocupados en el Río Bravo desde al menos 1904, aunque algunos de ellos llegaron dos décadas antes. Incluso desde este punto, varios se trasladaban a lugares tan distan- 
tes y mal comunicados como Nogales, Tijuana y el río Colorado, para atender las inspecciones y obras que se requerían, en las relaciones entre la SCOP y la CIL. Por otra parte, se ejecutaron las obras de defensa acostumbradas desde que las comenzaron Ignacio Garfias, Eduardo Prieto Basave, después Felipe Zavalza y luego Manuel María Mendiola, hasta que arribó a la ciudad el ingeniero Salvador Arroyo. Las obras continuaban concibiéndose de forma pasiva, se reforzaban de tiempo en tiempo los bordos que el estudio de los movimientos del río recomendaba, gracias a muchos trabajos de inspecciones y apoyos técnicos para la CIL. Una mirada al plano local indica que los ingenieros mencionados no solamente se asentaron en Ciudad Juárez para dirigir dichos trabajos, sino que, sobre todo, contribuyeron de forma importante a la organización del ramo de obras públicas en la elaboración de proyectos arquitectónicos, construcción de edificios e incluso - para el caso de Garfias y Mendiola- la elaboración de dos proyectos de planificación urbana, que se acompañaron de inspecciones y elaboración de dictámenes muy diversos para el Municipio (González Milea, 2021, pp. 40-72).

A lo largo de la década de 1890 se construyeron espolones en la ribera mexicana, con el fin de impedir la corrosión de la margen mexicana y defender a la ciudad de inundaciones. Pero lo cierto es que muchas obras protegían áreas con huertas. Con ocasión de las crecientes de 1891 se planteó un sistema de intervenciones más complejo, que incluía no solo los espolones, sino también terraplenes construidos con enfajinados de ramas y tierra, la arborización de ciertas áreas para reforzar el suelo y sobre todo varias obras de conexión de bordos y diques con el sistema de acequias de la ciudad, especialmente la más próxima al río, que se denominaba acequia del Chamizal (SCOP, 1900, p. 164). Las obras comenzaron a inicios de 1893, con gastos totales de 13 mil pesos, y continuaron año con año hasta 1896, cuando se erogaron casi 5 mil pesos en los trabajos (SCOP, 1900, p. 165), y solo hasta 1904 se publicó la estructura burocrática de la COD (SCOP, 1904, pp. 155-156, 162). 


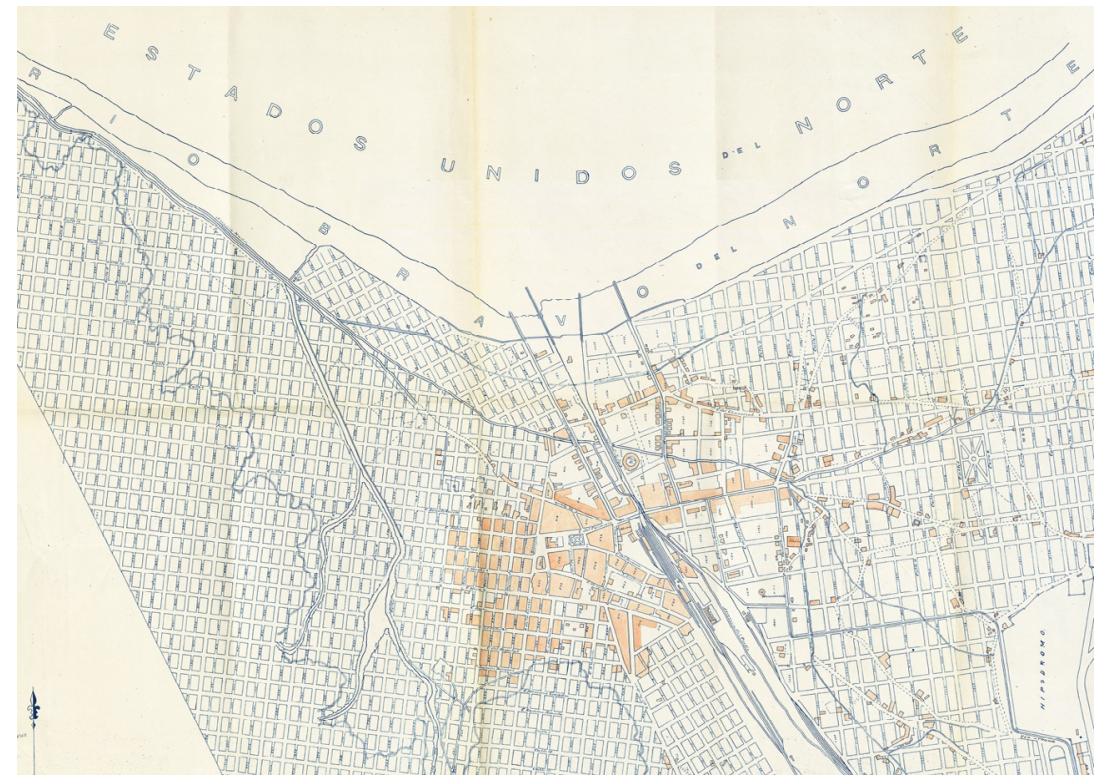

Figura 5. Este plan de urbanización fue elaborado por un técnico profundamente implicado en obras de defensa contra inundaciones. Plano de la ciudad moderna, elaborado por Manuel María Mendiola en 1905 y vuelto a imprimir en 1910 (AGN, SCOP, Ferr, Vol. 601).

El criterio de defensa en estos años sufrió un cambio importante. La idea, atribuida a Manuel María Mendiola, consistía en un verdadero muro que cubriría la ciudad a lo largo de su frente con el río: incluía en primer lugar un trabajo de hincado de 547 pilotes de pino creosotizado, cubriendo inicialmente una longitud de 330 metros para la cimentación del muro de piedra quebrada (más de 3 mil metros cúbicos); se añadían tirantes de madera y sujeciones de todos los pilotes. Al mismo tiempo, se levantaron nuevos mapas de las condiciones de toda la orilla en una longitud de cuatro kilómetros. Se gastaron en las obras un total de 38 mil pesos (SCOP, 1908, p. 248). Por otra parte, el criterio de la eliminación de bancos, como una forma práctica de equilibrar los terrenos perdidos y ganados en los movimientos del río, derivó en poco tiempo en la idea de que era posible rectificar el trayecto del río. Los estudios se habían practicado desde el año de 1905, 
eran coordinados desde la oficina de la CIL en Ciudad Juárez y se realizaron por medio de varias cuadrillas que se establecieron en campamentos temporales a todo lo largo del río hasta la ciudad de Matamoros.

En el periodo entre 1911 y 1912 la oficina en Ciudad Juárez adquirió el estatuto de dirección. Las crecientes del mes de julio habían destruido varias partes que todavía consistían en espolones y se procedió a repararlas conforme al mismo sistema, ahora descrito como un dique provisional formado de caballetes de madera que eran lastrados con piedra; claramente se trataba de la concepción de Garfias. Probablemente se emplearon los estudios que Mendiola había hecho unos años antes de los bancos de Bermúdez y San Lorenzo, donde se anunciaba la típica segregación que significaba terrenos nacionales perdidos porque el río podía tomar un nuevo curso; así se plantearon las obras provisionales en una longitud de ocho kilómetros. El monto ascendió a 16 mil pesos y el volumen a 102 pilotes de pino y 48 metros cúbicos de piedra (SCOP, 1912, p. 259). Sabemos, por otros datos, que en estos años Mendiola ya había salido de Ciudad Juárez y probablemente se hallaba cerca de la Sauteña, en Tamaulipas. Entre 1912 y 1921, las convulsiones de los cambios de régimen se apreciaban en la manera como los informes divagaban o esparcían información relativa a las obras, con ausencias importantes. Solamente hasta 1927 parece que se había logrado cierta estabilidad. En 1928, cuando el secretario era el ingeniero Ramón Ross, se informó que los trabajos que se realizaron durante los años de 1927 y parte de 1928 seguían teniendo el objetivo de dar mantenimiento a las obras de defensa, con las mismas técnicas, pero el gasto subió a 28 mil pesos (SCOP, 1928, p. 43). Es en estos años cuando se dio un viraje importante, pues en Ciudad Juárez comenzó a discutirse el proyecto de rectificación del río Bravo - mencionado en la memoria de 1929-, y en Matamoros el desarrollo de un sistema de contención de inundaciones, más bien con obras de canalización de excedentes.

Los técnicos de obras de defensa se involucraron en otros asuntos, como Salvador Arroyo, jefe de la COD en Ciudad Juárez, 
quien elaboró algunos comentarios respecto a la construcción de una garita en proximidad del bordo de defensa. Dado que conocía cómo estaba construido dicho bordo, dio algunos consejos sobre la manera de cimentar el pequeño edificio. En otro contexto, entre finales de 1926 e inicios de 1927, apoyó al gobierno de Chihuahua en los estudios para la construcción del nuevo edificio del Instituto Científico y Literario. ${ }^{30}$ El proyecto se había encargado al arquitecto Herny Trost, asentado en El Paso, Texas, pero los estudios que elaboró Arroyo contemplaron aspectos como abaratar el costo de construcción sin sacrificar los requerimientos espaciales e higiénicos. También elaboró unas fachada alternativas, todo probablemente a partir del proyecto del primer arquitecto. ${ }^{31}$ Por otro lado, a inicios de la década de 1930, el jefe de la COD era el ingeniero Alfonso Sáenz y en el Departamento de Edificios se desarrollaba un programa de obras para el mejoramiento de las oficinas federales en las ciudades fronterizas. El ingeniero Sáenz recopiló información sobre la manera y capacidad con que laboraba cada oficina federal en Ciudad Juárez y elaboró un programa arquitectónico. Al final, su propuesta de distribución espacial fue retomada por el ingeniero Argüelles para la elaboración de un proyecto de adaptación de la vieja aduana. ${ }^{32}$

30 AGN, CyOP, DE, Caja 103, Exp. 190072.

31 AGN, CyOP, DE, Caja 103, Exp. 190072.

32 AGN, CyOP, DE, Caja 55, Exp. 189709. 


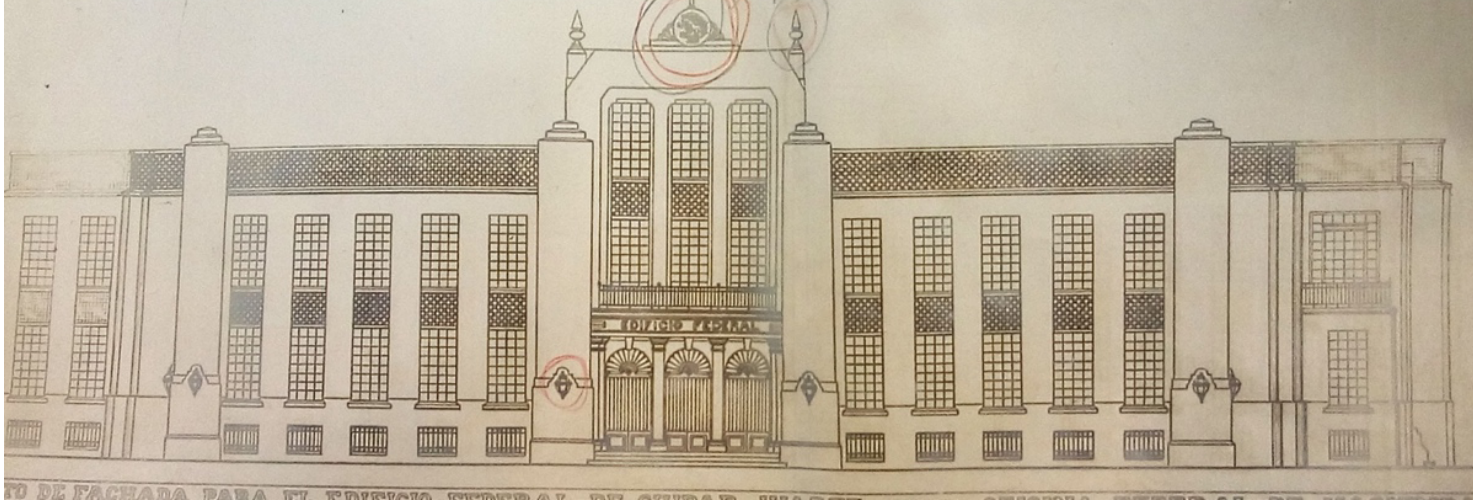

Figura 6. Elaborar dibujos y proyectos sobre edificios constituía un campo de saber presente entre muchos técnicos. Proyecto elaborado por los ingenieros de obras de defensa y José R. Argüelles, ca. 1930 (AGN, CyOP, DE, Caja 55, Exp. 189709).

En las décadas de 1930 y 1940 continuaron los trabajos de rectificación, hasta en 140 kilómetros, alternándose con obras de reforzamiento de bordos, construcción de canales interceptores e incluso nuevos monumentos para demarcación de la línea divisoria. A México le había tocado financiar solamente el 12\%, y en el plano práctico contó con el apoyo de la CNI (SCOP, 1937, p. 148; SCOP, 1939, p. 226; SCOP, 1940, p. 151; SCOP, 1943, pp. 78-79).

\section{LA DEFENSA EN LOS MEANDROS DEL BAJO RÍO BRAVO}

Como ya había señalado el ingeniero Mendiola desde 1896, la tercera sección del río era la más compleja, pues fluía con una pendiente suave, entre terrenos de constitución blanda dedicados al cultivo, esto provocaba figuras extremadamente sinuosas y frecuentes cambios del cauce y segregación de bancos. Si bien ya se habían ejecutado obras en Matamoros —en el puerto, pero también frente al Río Bravo - se trataba de intervenciones de índole pasiva, consistentes en la reparación de los viejos espolones siguiendo la técnica de Garfias (lanchas con motores de vapor instalados, martinetes, para hundir pilotes) (SCOP, 1900, p. 40). 
Aparecían urgencias, como la necesidad de rellenar brechas por alejamiento repentino del río (SCOP, 1904, p. 77; SCOP, 1908, p. 247), y en Matamoros se fraguaba un sistema de aprovechamiento de excesos de agua mediante el arroyo de Cabras Pintas (SCOP, 1928, p. 43). Hay que imaginar los grandes volúmenes y las duras condiciones del trabajo, pues las aguas debían canalizarse hasta el arroyo del Tigre a través de casi cuatro kilómetros, e incluso hubo que desaguar una laguna completa y desmontar a mano el terreno de casi 13 hectáreas (SCOP, 1929, p. 36; SCOP, 1937, p. 148; SCOP, 1939, p. 227; SCOP, 1940, p. 151; Walsh, 2008). En el caso de la pequeña localidad de Río Rico, frente a la población texana Thayer, todavía hasta la década de 1930 persistió la falta de criterios unitarios en las obras de defensa (cfr. Bustamante, 1999, p. 65). De acuerdo con el Tratado de Eliminación de Bancos, la pequeña localidad pasaría a los Estados Unidos - por tener menos de 250 habitantes-, pero el problema técnico implicaba la definición de dónde construir el bordo de defensa. ${ }^{33}$ Entre los años de 1934 y 1936 se incrementaron las tensiones entre los ingenieros de las obras de defensa -dependientes de la SCOP- y los técnicos de la CIL, respecto a la manera de concebir los proyectos en esta área de la ribera mexicana. Parece evidente que Chávez ocultó el proyecto del Retamal a los ingenieros de la CIL, pues desde junio de 1932 hasta agosto de 1937 no lo había mostrado todo por considerarlo innecesario (Melville, 2002) ${ }^{34}$ Quizás fue la única vez que un encargado de obras de defensa coordinó una serie de trabajos de índole completa en términos de planificación del desarrollo regional. Después de este episodio se intentaría distribuir las tareas técnicas entre la SCOP y la CIL para evitar conflictos. ${ }^{35}$

Al igual que en otros sitios de la frontera, no fue raro ver a los ingenieros de las obras de defensa desempeñando otras tareas que requería la SCOP. Fue lo que sucedió en Matamoros en 1933, cuando se encargó al ingeniero Aureliano T. Villaseñor, jefe de la sección de topografía, y a José María Ramírez, ingeniero en jefe

33 AHCILA, Gaveta 15, Exp. 1511.

34 AHCILA, Gaveta 32, Exp. 1824.

35 AGN, CyOP, DE, Caja 217, Exp. 208881. 
de la COD, que realizaran una inspección de los daños que había sufrido el edificio de la aduana por un reciente ciclón. ${ }^{36}$ En los siguientes años los ingenieros continuaron destacando la importancia de los entarquinamientos del suelo y, por lo tanto, de seguir el criterio de fortalecer de manera pasiva las riberas mexicanas del río, con cruces con enrocamientos y fajinas de jara y carrizo, e incluso la siembra de carrizos en grandes extensiones para evitar el movimiento o desplazamiento de las riberas. ${ }^{37}$

En Nuevo Laredo las cosas fueron distintas, pues de manera parecida a Ciudad Juárez, se juntaron los peligros por inundaciones con el crecimiento de la población. La ciudad en la primera mitad del siglo XX se fue convirtiendo en el cruce más importante de la frontera, ya que se constituyó en el sitio donde se recaudaba la mayor cantidad de impuestos a la importación. En la primera parte del siglo XX, un informe de la Dirección General de Bienes Nacionales indicaba que fueron las inundaciones de 1922 y 1932 las que obligaron a considerar un plan de trabajo, pues el agua había subido de nivel hasta 14 metros. Las construcciones eran de muy escasa calidad en la proximidad del río, por lo que convenía construir un bordo que al tiempo que tuviera suficiente solidez también ofreciera un aspecto agradable y atractivo como paseo; la corona del dique podría ser de hasta 12 metros de anchura, pavimentada y con parapeto hacia el lado del río, y estar arreglada con prados, árboles y bancas. Pero tal vez lo más importante era que se estaban considerando muchos tipos de obras "dentro de ese programa de mejoramiento de esta 'Puerta' de nuestro país” (Secretaría de Hacienda y Crédito Público [SHCP], 1938, p. 11). 
para "un pequeño museo tecnológico con su calificación". ${ }^{40}$ Pero también entre las décadas de 1930 y 1940 comenzó a hablarse de la necesidad de construir puertas de entrada al país en la frontera con Guatemala (SCOP, 1943, p. 79).

En Nuevo Laredo, por el contrario, el mismo encargo quedó a cargo de la oficina de bienes nacionales de la SHCP. ${ }^{41}$ Ya desde la década de 1923, con motivo de la concentración de las oficinas del gobierno federal en un solo edificio, el ingeniero Ignacio F. Lomelín hizo notar la dificultad de congeniar la necesidad de que estuviera situado en un punto cercano al cruce a los Estados Unidos y en un terreno a salvo de las inundaciones. ${ }^{42}$ Se imponía de tal modo el criterio de las obras de defensa. La memoria del SCOP de 1942 le dedicó un breve espacio al problema que recientemente se había desatado por las inundaciones de 1932 en Nuevo Laredo (SCOP, 1942, p. 103). Con anterioridad se había construido un puente nuevo, en 1922, pero resultaba inconveniente por la baja altura de sus arcadas; al mismo tiempo se construía el bordo de defensa, pero se avanzaba lento porque había que resolver las indemnizaciones. Aun así, los volúmenes de construcción de terracerías alcanzaron los $1200 \mathrm{~m}^{3}$, las demoliciones $15 \mathrm{mil} \mathrm{m}^{3}$ y los trabajos de consolidación $18 \mathrm{mil} \mathrm{m}^{3}$ (SCOP, 1943, p. 78). El gasto monetario y el volumen de los trabajos claramente se incrementaron en el siguiente periodo (SCOP, 1944, pp. 86-87) y continuaron vaciándose informes para el subsecuente, donde un balance general indicaba que se habían gastado en total en la obra poco más de 210 mil pesos (SCOP, 1945, p. 91).

En 1943, con motivo de los trabajos, un grupo de vecinos de la ciudad -el Comité Pro-Defensa contra Inundaciones del Río Bravo- se pronunció en contra de las características de las obras, en una misiva dirigida al secretario de la SCOP. Quizás con la asesoría del ingeniero Ricardo Treviño González, elaboraron una crítica sistemática conforme varios criterios y a fin de cuentas presentaron una propuesta de cómo debía concebirse el bordo

40 AGN, CyOP, DE, Caja 23, Exp. 19.

41 Un aspecto de la historia de las obras públicas que, sin duda, merecería varios estudios.

42 AGN, CyOP, DE, Caja 67, Exp. 189788. 
de defensa: apuntaban a problemas como su aspecto, los fines que debía cumplir la obra, las posibles consecuencias negativas e incluso su sentido patriótico. El primer punto concernía a la necesidad de construir algo más que un amontonamiento de tierra arenosa y buscar materiales más adecuados, sobre todo para dar una imagen decorosa y digna frente a los norteamericanos. En seguida se criticaba la posición de la obra, en vistas de la experiencia tenida, y se sugería extenderla hacia el poniente debido a que existía una vuelta pronunciada del cauce que podía significar una inundación. Otro criterio apuntaba a la forma en que de manera natural los vecinos sorteaban las dificultades de una inundación, dejando que al paso de 24 horas, o más, el nivel bajara por sí mismo. Otro era de índole climática, pues argumentaban los vecinos que el dique obstruiría la corriente de aire que hacía tolerables las noches de verano en la ciudad. ${ }^{43}$ El ingeniero de la CIL, Joaquín C. Bustamante, revisó la propuesta, pero la desechó, considerando que se trataba de un proyecto de hermoseamiento dado que no se presentaban planos de detalles técnicos.

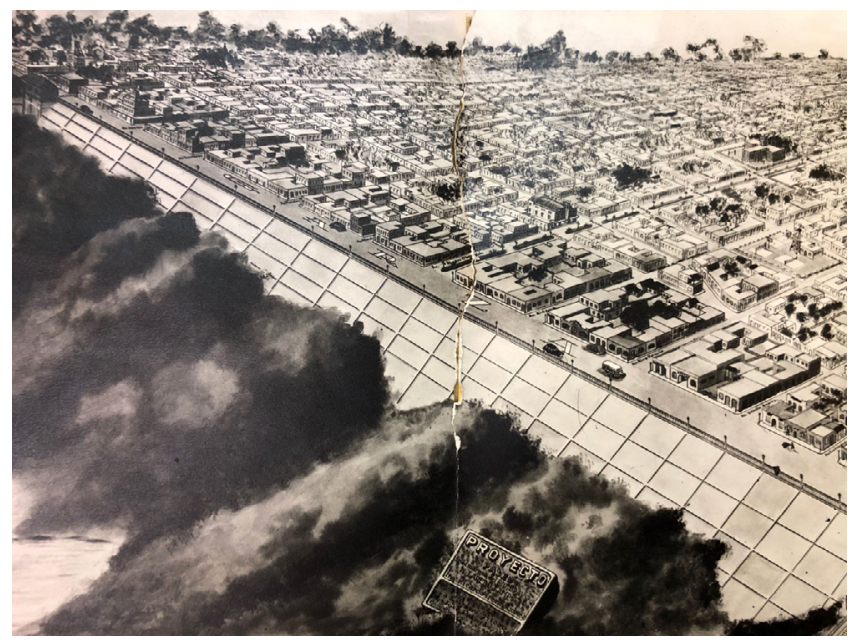

Figura 8. Propuesta de bordo por los vecinos de Nuevo Laredo, elaborado por el ingeniero Ricardo Treviño González (AHCILA, Gaveta 15, Exp. 1509).

43 AHCILA, Gaveta 15, Exp. 1509. 


\section{CONCLUSIONES}

En la frontera norte, a través de casi 100 años, el gobierno mexicano consiguió formular planes permanentes de obras públicas en el ámbito de la defensa contra inundaciones. A lo largo de este proceso se involucraron varias oficinas con competencias en la exploración e inspección del territorio y con capacidad para planear y ejecutar obras, en un momento en que las localidades fronterizas comenzaban a urbanizarse. La estructura administrativa de dichas oficinas, y de sus ministerios o secretarías de adscripción, también transitó por fases de reforma y periodos de convulsión política; pero aun así dichas oficinas fueron afianzándose en los territorios fronterizos, a través de un grupo de técnicos con trayectorias, formación profesional y ocupaciones muy heterogéneas. Al respecto, en este artículo se profundizó en la paulatina consolidación de la CIL y la COD, aunque también pudo apreciarse la injerencia de otras como la Comisión Nacional de Caminos y la Dirección de Bienes Nacionales. Todas confluyeron en las localidades fronterizas con el fin de elaborar dictámenes, e incluso para emprender trabajos de mayor envergadura. Si bien algunos estudiosos ya se han ocupado de los ambiciosos planes de la CNI -que engloban la llamada "gran hidráulica"-, lo cierto es que en el terreno de lo práctico abundaron "las diferencias de adscripción y criterio", incluso en el plano muy local de los ayuntamientos y comités de vecinos. Además, aunque las obras de defensa fueron el antecedente del manejo de cuencas fluviales, estas —con su espectro flexible y visión rudimentaria- no desaparecieron con la gran hidráulica. Esto ha quedado claramente ejemplificado a través de los estudios de Nogales, Ciudad Juárez y el Bajo Río Bravo.

Una ventaja de voltear la mirada a los trabajos de obras de defensa es que pueden advertirse tendencias distintas a las que se impusieron a las localidades bajo nociones de modernización. Si se examinara la historia de las profesiones se encontraría que, con anterioridad a la definición de perfiles específicos en las academias, en los sitios de trabajo los técnicos surcaban campos muy distintos de actividad; habría que sospechar del título con 
que se presentaban muchos ingenieros a través de las correspondencias. Muchas técnicas empleadas para definir la ubicación y consistencia de los bordos recuerdan los consejos de "engañar al río”, con que los tratados de arquitectura hidráulica del siglo XVIII aconsejaban enfrentar el peligro de inundaciones por crecientes.

Ciertamente, en la esfera de la administración, o burocracia técnica, la definición de ramos de obras públicas no fue un proceso continuo ni unificado y, como se vio, eran frecuentes los conflictos. Solo hasta 1944, en la SCOP se llevó a cabo una reforma administrativa de envergadura con el fin de erradicar "los paralelismos en obras públicas"; hasta entonces los trabajos eran encabezados por una oficina de obras hidráulicas que sirvió de nicho para una diversa y versátil burocracia técnica. Sus tareas reflejaron el estado de saberes prácticos antes que de ideales profesionales. Aun así, podría afirmarse que en este espacio socio-técnico convivieron topógrafos, hidrógrafos, astrónomos, peritos facultativos de minas, ingenieros civiles, ingenieros militares, arquitectos y varios practicantes solamente con estudios. La CIL, para hacer palpables los resultados de sus negociaciones diplomáticas, tuvo que bajar los argumentos al plano concreto del terreno mediante el brazo técnico de la COD.

En Nogales, las obras de canalización permitieron advertir a las autoridades locales que era necesario ordenar los permisos de edificación para establecer en qué sitios se podía construir y en cuáles no. Sobre todo, desde la introducción de las vías de ferrocarril se había definido lo que a través del tiempo se convertiría en el área central de la población, y al menos entre las décadas de 1930 y 1940 las decisiones del ingeniero José Argüelles y de los técnicos de la CIL tuvieron mucho peso. En Ciudad Juárez, el ingeniero Mendiola elaboró un plan de urbanización que contemplaba los requerimientos del riego y los peligros de inundación, aunque el peso de los regidores obligara a fin de cuentas a lotificar áreas que debían quedar libres. Lo cierto es que, el proyecto de rectificación del Río Bravo contribuyó a defender lo que al paso del tiempo se convertiría en área urbanizada y el posible desarrollo de un distrito de riego quedaría postergado de manera indefinida. 
Dado que la CIL se hallaba asentada en esta ciudad, no fue rara la influencia que tuvieron los ingenieros de la COD en otras partes de la frontera. Los demás ejemplos del también llamado Bajo Río Bravo sugieren la relevancia de las obras de defensa como parte del hermosamiento y planificación de ciudades. Sería interesante explorar los procesos locales en Nuevo Laredo y Matamoros para dimensionar la influencia de los técnicos de la SFCIC y de la SCOP en esas zonas.

\section{REFERENCIAS}

Archivos históricos

AGN. Archivo General de la Nación

CyOP. Comunicaciones y Obras Públicas

DE. Departamento de Edificios

LEUA. Límites con Estados Unidos de América

RyC. Ríos y Canales

Ferr. Ferrocarriles

AHHP. Archivo Histórico de Hidalgo del Parral

FSXIX. Fondo Siglo XIX

AHCILA. Archivo Histórico de la Comisión Internacional de Límites y Aguas

AHCJ. Archivo Histórico de Ciudad Juárez

PyT. Porfiriato y Terracismo

AHSRE. Archivo Histórico de la Secretaría de Relaciones Exteriores

LE. Legajo Encuadernado

Documentos institucionales

International Boundary Commission (IBC) (1932). Flood Control at Nogales, Arizona-Mexico, Letter from the Secretary of State to Senator Carl Hayden, from Arizona. Washington: U.S. Government Printing Office.

International Boundary and Water Commission (IBWC) (1938). Chronological Outline of the Organizations of the Various International Boundary and/or Water Commissions: Index to the 
Pertinent Treaties. Special Collections, University of Texas at El Paso.

Secretaría de Comunicaciones y Obras Públicas (SCOP) (1900). Memoria presentada al Congreso de la Unión por el Secretario de Estado y del Despacho de Comunicaciones y Obras Públicas de la República Mexicana, General Francisco Z. Mena, corresponde a los años transcurridos de $1^{\circ}$ de julio de 1891 a 30 de junio de 1896. Ciudad de México: Tipografía de la Dirección General de Telégrafos.

(1904). Anales de la Secretaría de Comunicaciones y Obras Públicas (Tercer Año, Primer Trimestre), (Enero). s.l.: s.p.i.

(1908). Memoria presentada al Congreso de la Unión por el Secretario de Estado y del Despacho de Comunicaciones y Obras Públicas de la República Mexicana, Ingeniero Leandro Fernández, corresponde al periodo transcurrido de $1^{\circ}$ de julio de 1906 a 30 de junio de 1907. Ciudad de México: Tipografía de la Dirección General de Telégrafos.

(1912). Memoria presentada al Congreso de la Unión por el Secretario de Estado y del Despacho de Comunicaciones y Obras Públicas de la República Mexicana, comprende el periodo transcurrido de $1^{\circ}$ de julio de 1911 a 30 de junio de 1912, Ingeniero David de la Fuente. Ciudad de México: Talleres Gráficos de la Secretaría de Comunicaciones y Obras Públicas.

(1928). Memoria presentada al Congreso de la Unión por el Secretario de Estado y del Despacho de Comunicaciones y Obras Públicas de la República Mexicana, comprende el periodo transcurrido de $2^{\circ}$ de agosto de 1927 al 31 de julio de 1928. Ciudad de México: Imprenta Galas.

(1929). Memoria presentada al Congreso de la Unión por el Secretario de Estado y del Despacho de Comunicaciones y Obras Públicas, comprende el periodo transcurrido del $2^{\circ}$ de agosto de 1927 al 31 de julio de 1928. México: Imprenta de Galas.

(1936). Memoria de los trabajos llevados a cabo por las dependencias de esta Secretaría durante el periodo de gobierno 
de septiembre de 1935 a agosto de 1936. Ciudad de México: Multigrafos SCOP.

(1937). Memoria de la Secretaría de Comunicaciones y Obras Públicas, septiembre de 1936-agosto de 1937, presentada al $H$. Congreso de la Unión por el C. General Francisco J. Mújica, Secretario del Ramo. Ciudad de México: D.A.P.P.

(1939). Memoria de la Secretaría de Comunicaciones y Obras Públicas, septiembre de 1938-agosto de 1939, presentada al H. Congreso de la Unión por el C. Secretario del Ramo Ing. Melquiades Angulo. Ciudad de México: D.A.P.P.

(1940). Memoria de la Secretaría de Comunicaciones y Obras Públicas, septiembre de 1939-agosto de 1940, presentada al H. Congreso de la Unión por el C. Secretario del Ramo Ing. Melquiades Angulo. Ciudad de México: s.p.i.

(1942). Memoria de la Secretaría de Comunicaciones y Obras Públicas (septiembre 1941-agosto 1942), presentada por el c. Secretario del Ramo, General de División Maximono Ávila Camacho. México: La Nacional Impresora S. A.

(1943). Memoria de la Secretaría de Comunicaciones y Obras Públicas, presentada por el C. Secretario del Ramo, General de División Maximino Ávila Camacho, septiembre 1942-agosto 1943. Ciudad de México: s.p.i.

(1944). Memoria de la Secretaría de Comunicaciones y Obras Públicas, presentada por el C. Secretario del Ramo, General de División Maximino Ávila Camacho, septiembre 1943-agosto 1944. Ciudad de México: s.p.i.

(1945). Memoria de la Secretaría de Comunicaciones y Obras Públicas, presentada por el c. Secretario del Ramo, Ing. Pedro Martínez Tornel, septiembre 1944-agosto 1945. México: s.p.i.

Secretaría de Fomento, Colonización, Industria y Comercio (SFCIC) (1857). Memoria de la Secretaría de Estado y del Despacho de Fomento, Colonización, Industria y Comercio de la República Mexicana, escrita por el Ministro del Ramo, D. Manuel Siliceo, para dar cuenta con ella al Soberano Congreso Constitucional (Parte I). Ciudad de México: Imprenta de Vicente García Torres. 
(1877). Memoria presentada al Congreso de la Unión por el Secretario de Estado y del Despacho de Fomento, Colonización, Industria y Comercio de la República Mexicana, Vicente Riva Palacio. Ciudad de México: Imprenta de Francisco Díaz de León.

(1885). Memoria presentada al Congreso de la Unión, por el Secretario de Estado y del Despacho de Fomento, Colonización, Industria y Comercio de la República Mexicana (Tomos 1, 2 y 3). Ciudad de México: Oficina Tipográfica de la Secretaría de Fomento.

(1887). Memoria presentada al Congreso de la Unión, por el Secretario de Estado y del Despacho de Fomento, Colonización, Industria y Comercio de la República Mexicana (Tomos 1, 2, 3 y 5). Ciudad de México: Oficina Tipográfica de la Secretaría de Fomento.

Secretaría de Hacienda y Crédito Público (SHCP) (1938). Planificación de Nuevo Laredo, Ciudad de México: SHCP/Dirección General de Bienes Nacionales/D. A. P. P.

Secretaría de Relaciones Exteriores (SRE) (1896). Memoria de la Comisión Internacional de Límites, México. AGN, Límites con Estados Unidos de América, Caja 3, Legajo 4, Exp.105.

(1901). Memoria de la Sección Mexicana de la Comisión Internacional de Límites entre México y los Estados Unidos de América, que restableció los monumentos de El Paso al Pacífico, durante los años de 1892 a 1894, Nueva York, U.S.A. AHSRE, LE-2255.

(1903). Proceedings of the International (Water) Boundary Commission (Volume 2). Washington: U. S. Government Printing Office.

(s.f.). Tratados y Convenciones sobre Límites y Aguas entre México y los Estados Unidos, Ciudad Juárez. 


\section{BIBLIOGRAFÍA}

Aboites Aguilar, L. (1987). La irrigación revolucionaria. Ciudad de México: Secretaría de Educación Pública.

Aguilar Civera, I. (2012). El discurso del ingeniero en el siglo XIX: Aportaciones a la historia de las obras públicas. Madrid: Fundación Juanelo Turriano/Generalitat Valenciana.

Álvarez, M. F. (1906). El Dr. Cavallari y la carrera de ingeniero civil en México. Ciudad de México: A. Carranza y Comp. Impresores.

Álvarez, C. J. (2019a). Border Land, Border Water: A History of Construction on the US-Mexico Divide. Austin: University of Texas Press.

Álvarez, C. J. (2019b). Police, Waterworks, and the Construction of the U.S.-Mexico Border, 1924-1954. Western Historical Quarterly, 50(3), 233-256.

Anand, N., Gupta, A. y Appel, H. (2018). Introduction: Temporality, Politics, and the Promise of Infrastructure. En N. Anand, A. Gupta y H. Appel (Eds.), The Promise of Infrastructure (pp. 1-38). Durham y Londres: Duke University Press.

Bustamante Redondo, J. (1999). La Comisión Internacional de Límites y Aguas entre México y los Estados Unidos. Ciudad Juárez: Universidad Autónoma de Ciudad Juárez.

Castro, J. J. (2019). Apostle of Progress: Modesto C. Rolland, Global Progressivism, and the Engineering of Revolutionary Mexico. Lincoln y Londres: University of Nebraska Press.

Connolly, P. (1999). Introducción. En S. Kuntz y P. Connolly (Coords.), Ferrocarriles y Obras Públicas (pp. 141-164). Ciudad de México: Instituto Mora/Colegio de Michoacán/El Colegio de México/Universidad Nacional Autónoma de México.

Davis, H. (2016). The Culture of Building. Nueva York: Oxford University Press.

González de la Vara, M. (2017). Región, frontera y capitales: Inversiones, política fronteriza y cambio socioeconómico en la región binacional de El Paso-Ciudad Juárez, 1846-1911. Zamora: El Colegio de Michoacán/El Colegio de la Frontera Norte/ 
Universidad Autónoma de Ciudad Juárez/El Colegio de Chihuahua.

González Milea, A. (2021). Una mirada a los constructores de una ciudad fronteriza: Ingenieros, arquitectos y maestros de obra en Ciudad Juárez (1888-1928). Ciudad Juárez: Universidad Autónoma de Ciudad Juárez/Universidad Autónoma de Chihua-hua/Secretaría de Cultura-Gobierno de Chihuahua.

Larkin, B. (2013). The Politics and Poetics of Infrastructure. Annual Review of Anthropology, 42, 327-343.

Melville, R. (2002). Las obras de defensa del bajo Río Bravo: Antecedente histórico en el manejo de cuencas fluviales. En P. Ávila García (Ed.), Agua, cultura y sociedad (pp. 199-207). Zamora: El Colegio de Michoacán/Instituto Mexicano de Tecnología del Agua.

Samaniego, M. A. (2006). Ríos internacionales entre México y EStados Unidos: Los tratados de 1906 y 1944. Ciudad de México: El Colegio de México/Universidad Autónoma de Baja California.

Tinker Salas, M. (2001). Los dos Nogales. En M. C. Ceballos (Coord.), Encuentro en la frontera: Mexicanos y norteamericanos en un espacio común (pp. 259-279). Ciudad de México: El Colegio de México/El Colegio de la Frontera Norte/Universidad Autónoma de Tamaulipas.

Walsh, C. (2008). Building the Borderlands. A Transnational History of Irrigated Cotton along the Mexico-Texas Border. College Station: Texas A\&M University Press.

Walsh, C. (2012). Mexican water studies in the Mexico-US borderlands. Journal of Political Ecology, 19(1), 50-56. 